\title{
The Analysis of Influence of Throttle Body on Engine Intake System
}

\author{
ChangChun $\mathrm{Xu}^{\# 1}$, HaengMuk $\mathrm{Cho}^{* 2}$ \\ \#Division of Mechanical and Automotive Engineering, Kongju National University 1223-24, \\ Cheonan Daero, Seobuk-gu, Cheonan-si, Chungcheongnam-do 331-717, South Korea. \\ ${ }^{1}$ xccgh78006@naver.com \\ *Division of Mechanical and Automotive Engineering, Kongju National University 1223-24, \\ Cheonan Daero, Seobuk-gu, Cheonan-si, Chungcheongnam-do 331-717, South Korea. \\ 2hmcho@kongju.ac.kr
}

\begin{abstract}
At present, the car is the normal means of transport in people's homes. However, the car to the people to bring convenience, the environment caused a variety of pollution, including emissions and noise pollution. This paper focuses on optimizing the disc angle, finding a better angle, reducing noise, and reducing exhaust. When the car is running, the air enters the engine before the air through the throttle body, because the different angles of the butterfly plate, respectively, led to the throttle body flow and flow pressure is different. This result can cause noise due to the difference in flow rate and flow pressure. And different speeds and pressures may cause a different mixture of air and fuel in the engine. Thus causing the A / F ratio in the cylinder to be different, resulting in the mixing of the air fuel (A / F) too thick or too thin when the engine is burned. Incomplete combustion led to the occurrence of exhaust, can pollute the human living environment. In order to make the car comfortable and no noise, in order to make air and fuel in the engine completely burn, the author can adjust the throttle to achieve this result. Therefore, in this paper, the author can simulate the turbulence program to find the optimal installation of the throttle body to reduce noise and exhaust emissions. The authors change the angle of the throttle body, such as 0 degrees, 30 degrees, 45 degrees, 60 degrees and 90 degrees. In these angles, the authors can compare these angles at specific locations to compare velocity and pressure and flow distributions to find the optimum angle for engine combustion at 60 degrees.
\end{abstract}

Keyword - Throttle body, Noise reduction, Exhaust emission, Turbulence, Combustion.

\section{INTRODUCTION}

In this paper, authors analyze the mixing ratio of air and fuel into the engine cylinder by changing the angle of the throttle valve, that is, by changing the angle of the throttle and thus changing the amount of air entering the cylinder, authors can find Optimize throttle angle that improving the exhaust emissions.

Main function of a throttle body assembly is to control the air flow into the engine based on vehicle demand. Throttle body is mounted between the air cleaner and the intake manifold. Following the angle of throttle valve changes, butterfly valve restricts the amount of airflow into the engine cylinder.

In this analysis process, authors set the butterfly valve angle to $0,25,50,75,100 \%$ throttle opening conditions. At these conditions authors can find the Optimize throttle angle for air and fuel ratio into the engine cylinder. In this study, three types of boundaries are involved including inlet, outlet, and wall. Inlet pressure boundary conditions are used to set the fluid pressure at the flow inlet. Therefore authors can set the pressure when the pressure is not known.

And outlet boundary conditions require the specification of static pressure at the outlet boundary. With the air flow inlet through the throttle valve, authors can analysis the counter and vector of air stream in the different angles. Authors can find the effects of throttle valve opening on flow field in order to see the wake regions and velocity vectors in this analysis.

\section{EXPERIMENT ANALYSIS}

In order to compare the different results of the experiment by using CFD program, authors set the experiment use the Optimization shape that Hexagonal shape throttle valve to analyze the air flow through throttle body into the engine cylinder. The flow rate of the air is adjusted by flow control valve and measured with the help of CFD. With the simulation of the throttle body, the experiment can show us the air flow image and fluid velocity, and initially the throttle opening is kept corresponding to engine idling conditions. The test pressure is set and flow through the throttle body is measured. Therefore authors set the angle for $25,50,75$, and $100 \%$ throttle opening positions flow rates can be measured. During in these experiments, with find optimization angle of the throttle body for air and fuel equivalent ratio and engine speed, the exhaust emissions (consist of HC, CO, $\mathrm{CO}_{2}$ ) can be measured. 
In this experiment process, in order to realize exhaust emission to reduce, and for reducing the environmental pollution of gas exhaust, authors set the throttle valve angle for 0, 30, 45, 60, 90 degrees respectively to compare. With the pressure and velocity compare, find the angle of the throttle valve for optimization of exhaust emission.

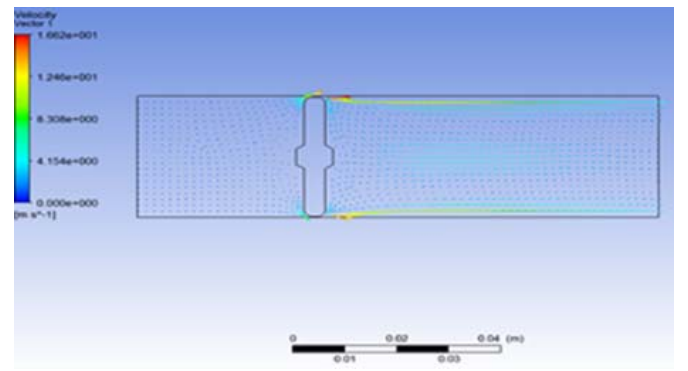

0 degrees

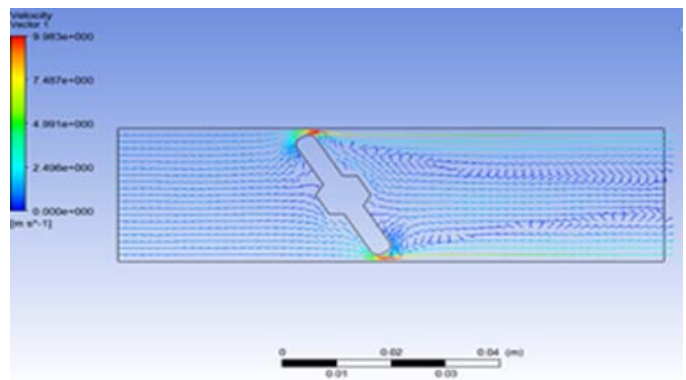

30 degrees

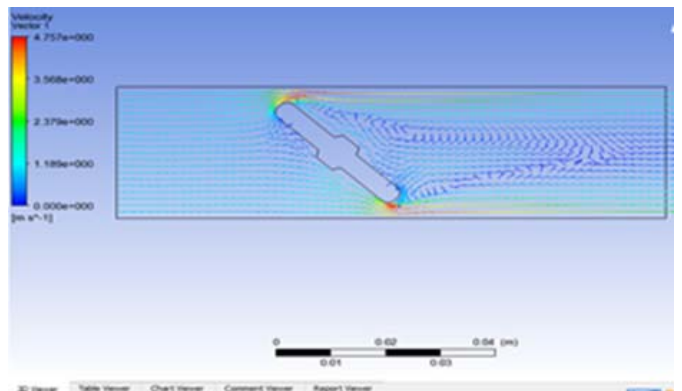

45 degrees

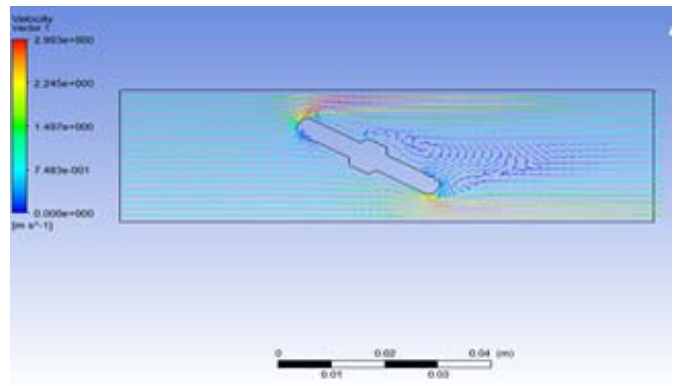

60 degrees

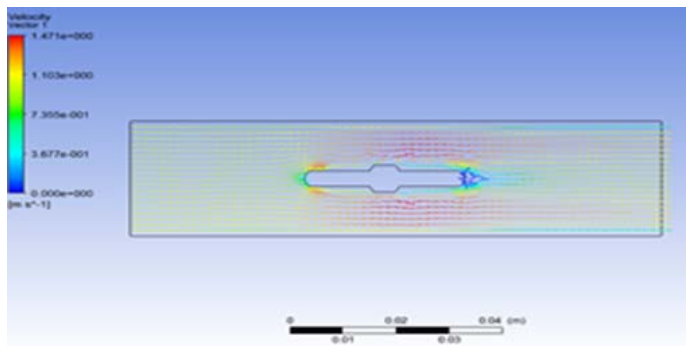

90 degrees

Fig. 1 Velocity plots of the different throttle opening positions 
As can be seen from Figure 1, the velocity of the different throttle opening positions indicates the eddy current of the airflow at the $30^{\circ}, 45^{\circ}$ and $60^{\circ}$ angle of the throttle opening through the flow velocity of the throttle valve. In order to introduce air into the engine cylinder at a moderate speed, the vortex area cannot be too large, otherwise it will cause the air and fuel mixture to be uneven. If the air and fuel mix is not good, it may cause HC, $\mathrm{CO}$ exhaust emissions. Therefore, in order to discharge exhaust and air and fuel mixture, the author believes that the angle of the throttle opening is 60 degrees.

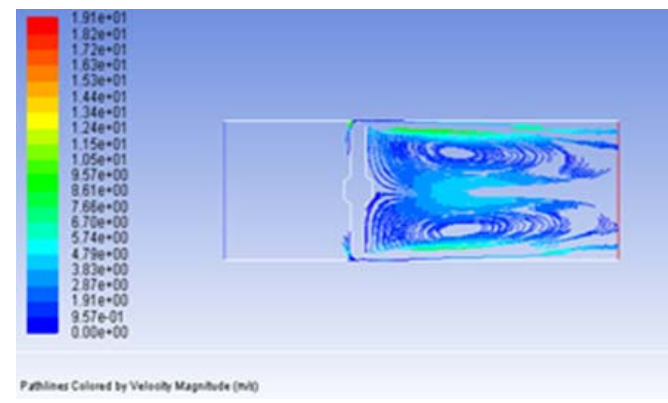

0 degrees

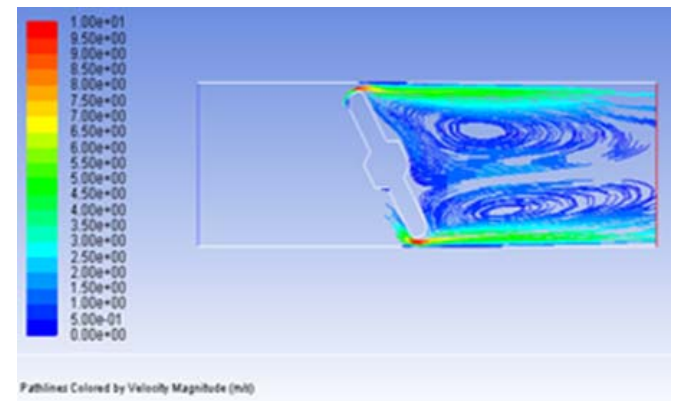

30 degrees

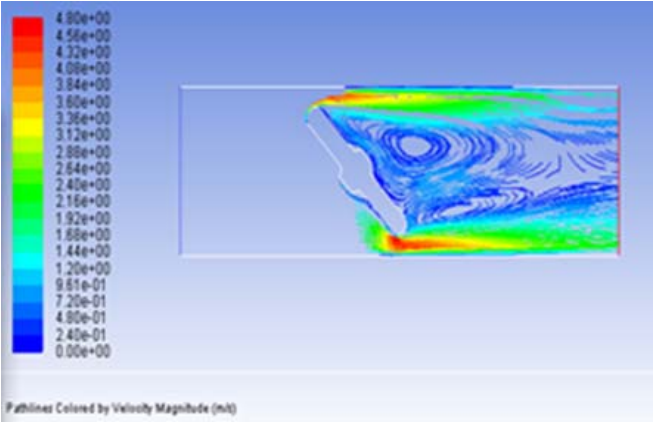

45 degrees

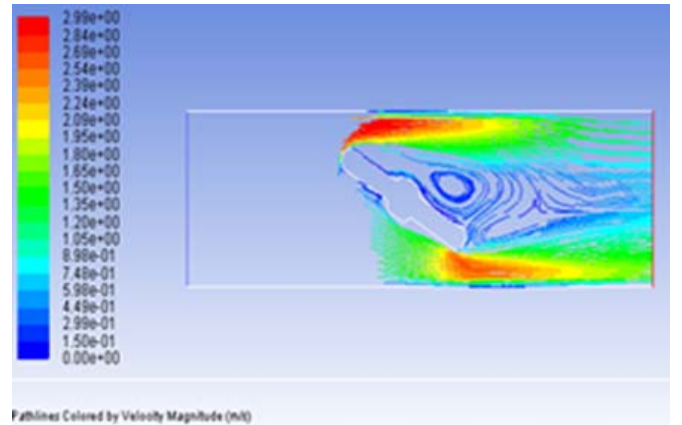

60 degrees 


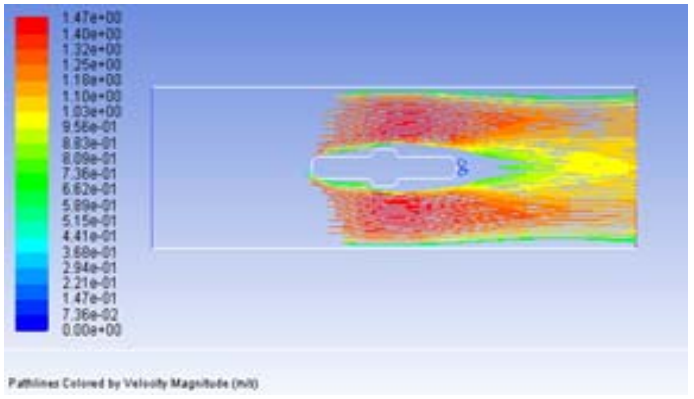

\section{0 degrees}

Fig.2 Flow distribution of velocity of the different throttle opening positions

From the above Figure 2, the speed distribution of the different throttle opening position, when the angle is 0,30,45 degrees, you can find the resulting wake area, resulting in air into the engine is very rare, resulting in uneven mixing of air and fuel, Resulting in incomplete oxidation of the product. Although the 60-degree flow distribution, there will be a wake area, but after a short period of time will soon return to normal. The authors believe that the impact of air-fuel combination is not. So the author believes that the throttle of 60 degrees is A / F mixed with better conditions.
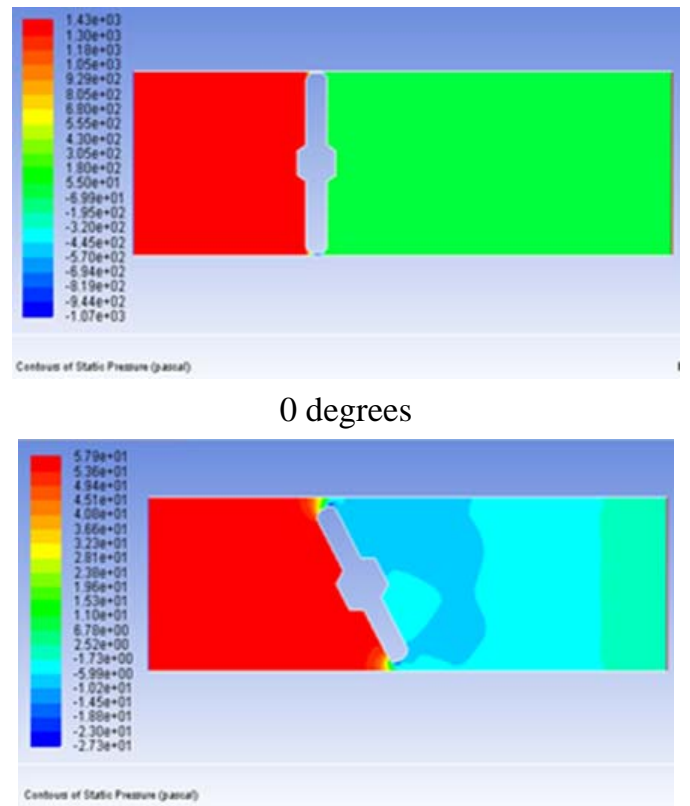

30 degrees

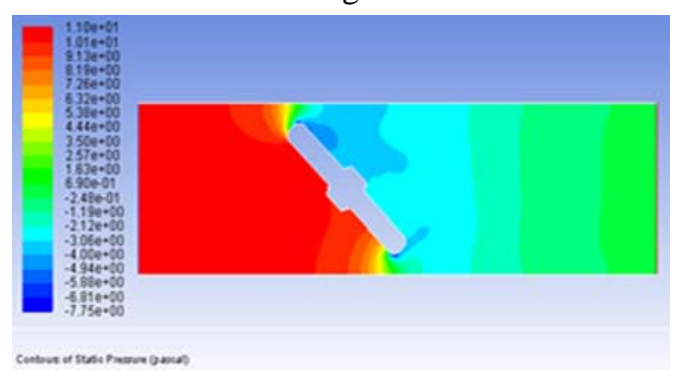

45 degrees 


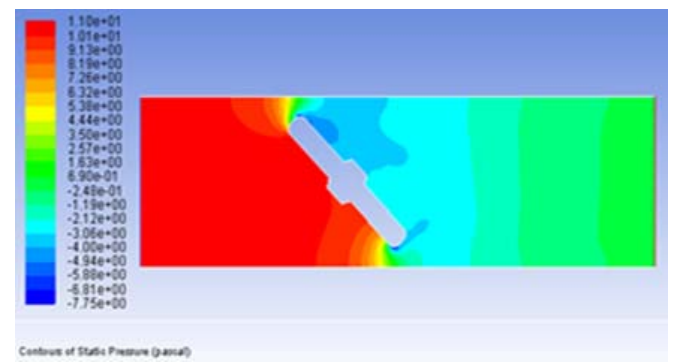

60 degrees

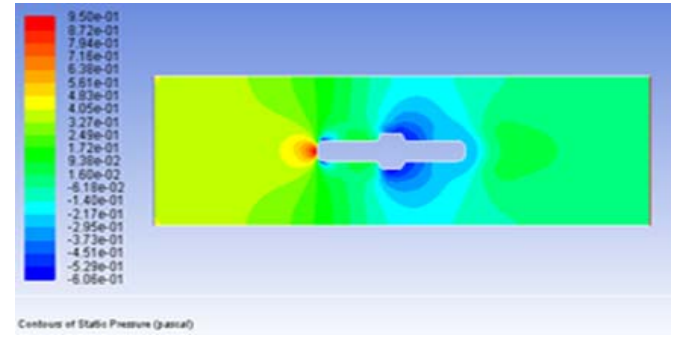

90 degrees

Fig. 3 Pressure plots of the different throttle opening positions

It can be seen from Figure 3, the different throttle opening position of the pressure curve shows the throttle opening is $0,30,45,60,90$ degrees. Due to the relationship between velocity and pressure, the pressure of the regional fluid velocity is slow and the pressure of the small area fluid velocity will be accelerated. The above figure shows our pressure distribution, in order to make the air and fuel mixed evenly, so the air into the engine at low speed, the export air area to make the pressure larger. So, the author can compare these images to find 0 degrees, 45 degrees and 60 degrees of the throttle valve is a good angle for the air flow into the engine. Because 0 degrees without air through the throttle lead to energy waste and idle time will also lead to exhaust. On the other hand, in view of these angles from the throttle opening position in order to reduce airflow noise, these images show that the throttle opening position for reducing airflow noise is 60 degrees and 90 degrees.

Synthesis, the two aspects of the experimental comparison, the author believes that the best angle of the throttle opening degree of 60 degrees. As the angel can make the airflow outlet pressure larger, above the throttle valve, you can see the negative pressure area, these areas can make the air vortex produce lower airflow energy, reduce noise.

\section{III.CONCLUSION}

Through the turbulence system you can see that the different throttle opening positions are $0,30,45,60$ and 90 degrees. By studying the angle of the throttle opening position, in order to compare the different angles, the air velocity distribution and the pressure distribution found that the better position of the throttle opening position was 60 degrees.

Not only to mix air and fuel evenly, but also found that 60 degrees can be reduced to the engine cylinder noise, due to the negative pressure area, so that air energy is reduced, so that the speed through the exit.

Comprehensive pressure experiment, through these experiments, the author believes that the best angle of the throttle opening is 60 degrees. As the angel can make the airflow outlet pressure larger, above the throttle valve, you can see the negative pressure area, these areas can make the air vortex produce lower airflow energy, reduce noise.

In this paper, the angle of the throttle opening position when the air flows through the throttle valve is compared to find the optimum angle of the throttle valve for reducing the exhaust gas, since the throttle body can control the flow of air into the engine cylinder and the fuel Equivalent ratio), control of air and fuel fully mixed HC, CO exhaust reduction, reduce noise and vibration.

\section{ACKNOWLEDGMENT}

This research was supported by The Leading Human Resource Training Program of Regional Neo Industry through the National Research Foundation of Korea(NRF) funded by the Ministry of Science, ICT and future Planning(NRF-2016H1D5A1909917) 


\section{REFERENCES}

[1] J.Suresh Kumar ${ }^{\mathrm{a}}$, V.Ganesan ${ }^{\mathrm{a}}$, J.M.Mallikarjuna ${ }^{\mathrm{a}}$ \& S.Govindarajan ${ }^{\mathrm{b}}$, Design and optimization of a throttle body assembly by CFD analysis, India Journal of Engineering \& Materials Sciences, Vol. 20 October 2013, pp.350-360.

[2] Kriti Gupta, Saumya Sharma, Jayashree Aseri, Anupriya, CFD Analysis of Flow through a Throttle Body of a Spark Ignition Engine for diffenent Throttle Valve Shaft Configurations, International Journal of Engineering and Technical Research(IJETR) ISSN: 23210869(O)2454-4698(P), Volume-5,Issue-4, August 2016.

[3] Dr.Alexander Morozov, AVL List GmbH, A-8020 Graz, Dr.Uwe Iben, Robert Bosch GmbH, Dept. CR/ARH, D-70049 Stuttgart, Experimental Analysis and Simulation of Cavitating Throttle Flow, HEFAT 2008,30 June to 2 July 2008, Pretoria, South Africa.

[4] Kazuhiko Ogawa, Noise Reduction in Butterfly Valve Cavitation by Semicircular Fins and Visualization of Cavitation Flow. Osaka Sangyo University, Japan.

[5] Mustapha Bordjane, David Chalet, Numerical Investigation of Throttle Valve Flow Characteristics For Internal Combustion Engines, Journal of Multidisciplinary Engineering Science and Technology(JMEST) ISSN:3159-0040, Vol.2 Issue12, December-2015.

[6] Mehmet SANDALCI ${ }^{1}$, Ebru MANCUHAN ${ }^{2}$, Emre ALPMAN ${ }^{2}$, Kurtul KUCUKADA ${ }^{3}$, Effect of the Flow Conditions and Valve Size on Butterfly Valve Performance, J. of Thermal Science and Technology, Isi Bilimi ve Teknigi Dergisi, 30,2, 103-112,2010.

[7] Farid Vakili-Tahami ${ }^{1}$, Mohammad Zehsaz ${ }^{1, *}$, Mahdi Mohammadpour ${ }^{2}$ and Ali Vakili-Tahami ${ }^{3}$, Analysis of the hydrodynamic torque effects on large size butterfly valves and comparing results with AWWA C504 standard recommendations, Journal of Mechanical Science and Technology 26 (9) (2012) 2799-2806.

[8] Se Youl Won, Jae Gon Lee, Jun Seok Yang, The Effect of the Variation of the Downstream Region Distance and Butterfly Valve Angle on Flow Characteristics in a 90 Degree Bended Elbow, Modern Mechanical Engineering, 2014,4,133-143.

[9] Ronald G. Huff, Lewis Research Center, Cleveland, Ohio, Noise Generated by Flow Through Large Butterfly Valves, NASA Technical Memorandum 88911, January 1987.

\section{AUTHOR PROFILE}

ChangChun Xu, Ph.D. student of Professor Cho. Study in the Department of Mechanical Engineering, Kongju National Unversity, South Korea.

HaengMuk Cho, Ph.D. Experienced in R\&D of internal combustion engines and advanced turbo technologies, alternate energy with the knowledge of advanced engineering/scientific techniques. Expert in 3D design in SolidWorks and CFD simulation. Member of The Korean Society of Mechanical Engineers. 